\title{
Simultaneous X-ray/radio observations of Cir X-1
}

\section{Paolo Soleri*}

Astronomical Institute "A. Pannekoek", University of Amsterdam, Kruislaan 403, NL-1098 SJ,

Amsterdam, The Netherlands

E-mail: psoleriescience.uva.n]

\section{Valeriu Tudose}

Astronomical Institute "A. Pannekoek", University of Amsterdam, Kruislaan 403, NL-1098 SJ,

Amsterdam, The Netherlands

E-mail: vtudose@science.uva.nl

\section{Rob Fender}

School of Physics and Astronomy, University of Southampton, Highfield, Southampton SO17 1BJ

E-mail: rpfephys.soton.ac.uk

\section{Michiel van der Klis}

Astronomical Institute "A. Pannekoek", University of Amsterdam, Kruislaan 403, NL-1098 SJ,

Amsterdam, The Netherlands

E-mail: michiel@science.uva.nl

We analysed simultaneous X-ray/radio observations of Circinus X-1 collected respectively with RXTE and ATCA in 2000 October and 2002 December and identified radio flares close to phase 0.0 and 0.5 of the orbital period. To date, there is only circumstantial evidence for radio flares near phase 0.5. Moreover, in our data set, we clearly associated both a radio flare and X-ray spectral timing changes with phase 0.0 . While for black hole X-ray binaries the picture of the association between the X-ray and the radio bands is quite well understood, for neutron star X-ray binaries a clear and complete picture is still missing.

Bursts, Pulses and Flickering:Wide-field monitoring of the dynamic radio sky June 12-15 2007 Kerastari, Tripolis, Greece

\footnotetext{
* Speaker.
} 


\section{Introduction}

Low magnetic field neutron star X-ray binaries (NSXBs) are often divided in two classes, according to their correlated spectral and timing properties: the "atoll" and the "Z" sources [13], named after the shape of the track they draw in the X-ray color-color diagrams (CDs) and the hardness-intensity diagrams (HIDs).

$\mathrm{Z}$ sources are brighter than atoll sources and are believed to accrete at near-Eddington luminosities (0.5-1.0 $L_{E d d}$, [34]). To date seven Galactic NSXBs have been classified as Z sources: six of them are persistent, one of them is transient (XTE J1701-462, [16]; as the source faded to quiescence it switched to atoll-type on the way down, [17]). They are characterized by three-branched tracks in their CDs and HIDs that in some cases resemble the character " $\mathrm{Z}$ ". The three branches are called horizontal branch (HB), normal branch (NB) and flaring branch (FB). The mass accretion rate, $\dot{m}$, is assumed to drive the transitions between the branches, increasing monotonically from the $\mathrm{HB}$ to the FB, affecting both the spectral and the timing properties.

Power density spectra (PDS) of the Z sources show several types of quasi periodic oscillations (QPOs) and noise components [34], whose presence and properties are strongly correlated with the position of the source along the $Z$ track [13]. Three types of low frequency QPOs $(<100 \mathrm{~Hz})$ are seen in the $Z$ sources, each of them usually detected in just one of the three branches (but see [35], [36]); twin kHz QPOs have also been detected in all the $\mathrm{Z}$ sources (only marginally detected in XTE J1701-462, [16]). Other characteristic noise components of $Z$ sources are the very low frequency noise (VLFN) and the low frequency noise (LFN).

All the Z-type NS sources are detected in the radio band, showing large and rapid variability, optically thin and optically thick emission. In 1998 it has been found for the first time (in GX 17+2) that the radio emission varies as a function of the position in the X-ray CD [27], decreasing with increasing mass accretion rate from the HB (strongest radio emission) to the FB (weakest radio emission). Recently it has been suggested suggested that this behaviour could be universal [21] (but see [31] for GX 5-1). Extended radio jets have been spatially resolved for Sco X-1 and have also been associated to ultra-relativistic ejections [11].

\subsection{Cir X-1}

Cir X-1 was discovered in 1971 [20] and has been showing flares with a period of 16.55 days, observed first in the X-ray band [19] and then in the infrared [12], radio [14] and optical bands [22]: this fact is interpreted as enhanced accretion close to the periastron passage of a highly eccentric binary orbit $(e \sim 0.8,[23$, [24]). The source is located in the galactic plane at a distance that has been reported to lie in the range 4-12 kpc (see [18] for a recent discussion). Many properties of Cir X-1 would suggest that this is a black hole candidate (BHC): it has strong radio emission (e.g. [14]), ultra-relativistic radio jets (the most relativistic detected so far within our galaxy, [9]), hard $\mathrm{X}$-ray emission [6] and very strong X-ray variability [26]. The first strong indication that the binary system harbours a neutron star has been reported in 1986 [32], when type-I X-ray bursts have been detected in EXOSAT data. After [32] no type-I X-ray bursts have been reported. Shirey et al, in an extensive analysis of RXTE/PCA data, identified typical low-frequency Z-source features in Cir X-1 PDS [29] and then a complete Z track in its high-luminosity orbital phases [30]. In 1999 it has been noted that the characteristic timing frequencies of Cir X-1 lie in between those typically 
associated with NS and BH systems [28]. Recently twin $\mathrm{kHz}$ QPOs have been reported for the first time in Cir X-1 PDS [3], a further indication of the nature of the compact object.

Cir X-1 lies within a radio nebula [33] and shows radio jets that have been spatially resolved [8]; the radio nebula is produced by synchrotron emission likely originating in the interaction between the jet and the interstellar medium. Recently evidence of an extended arcmin-scale X-ray jet around the source has been reported [15], in the same direction as the receding radio jet, making Cir X-1 the first secure neutron star system for which an extended X-ray jet has been resolved.

\section{Observations and data analysis}

\subsection{X-ray data}

We analysed 22 RXTE/PCA observations made between 2000 October 1 and 2000 October 26 and 23 RXTE/PCA observations performed between 2002 December 3 and 2002 December 10.

Background subtracted light curves with a time resolution of 16 seconds were obtained from the "Standard 2"-mode data, covering the energy range 2-18 keV and dead-time corrections were applied. We defined two X-ray colors, a hard color (HC) and a broad color (BC), in the following energy bands: (8.5-13)/(13-18) $\mathrm{keV}$ (HC) and (2-6.3)/(6.3-13) $\mathrm{keV}(\mathrm{BC})$.

In addition, for each 128-second time interval, we accumulated power density spectra in the $\sim 2-33$ $\mathrm{keV}$ and $\sim 2-10 \mathrm{keV}$ energy ranges (respectively for 2000 October and 2002 December), with a Nyquist frequency of $8192 \mathrm{~Hz}$. Further details on the data analysis will be given in a future paper (Soleri et al., in preparation).

New radio ephemeris (determined in 2007) was used to calculate the orbital phases [25].

\subsection{Radio data}

We have observed Cir X-1 in radio (simultaneously with X-ray) over multiple epochs on 2000 October and 2002 December at 4.8 and $8.6 \mathrm{GHz}$ using the Australia Telescope Compact Array (ATCA). We used PKS J1939-6342 (PKS B1934-638) as primary calibrator and PMN J1524-5903 as secondary calibrator (B1520-58).

\section{Results}

Figure 1 shows the RXTE/PCA light curves of Cir X-1 for our data set: in 2000 October more than an entire orbit was covered, albeit sparsely, while in 2002 December the data were focused around periastron passage (phase 0.0 of the orbital period). Since a detailed discussion about the evolution of the X-ray/radio properties in the whole analysed data set is beyond the scope of this paper, for now we will focus our attention on two particular orbital phases where we noticed remarkable behaviour. An extensive discussion of the X-ray and the correlated X-ray/radio properties of this data set will be presented in a future paper (Soleri et al., in preparation).

\subsection{December - Phase 0.0 of the orbital period}

Figure 2 shows the radio light curve, the X-ray light curve and the X-ray hardness curve for the orbital phase interval $0.99-1.15$, in 2002 December. After the passage through phase 0.0 (but 


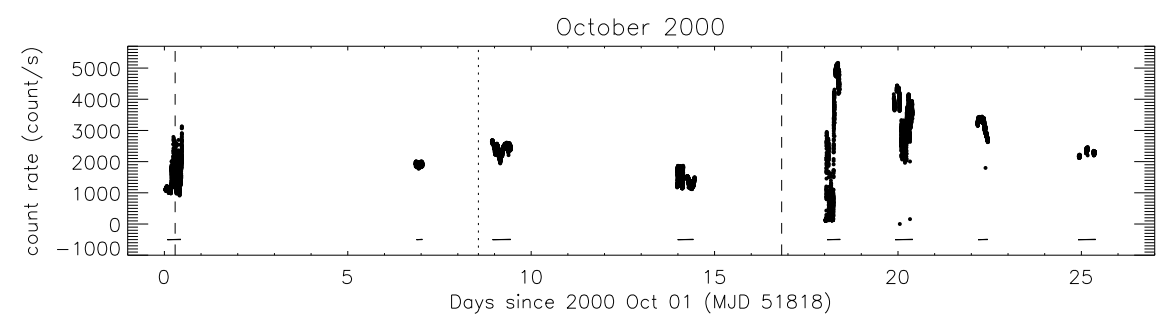

December 2002

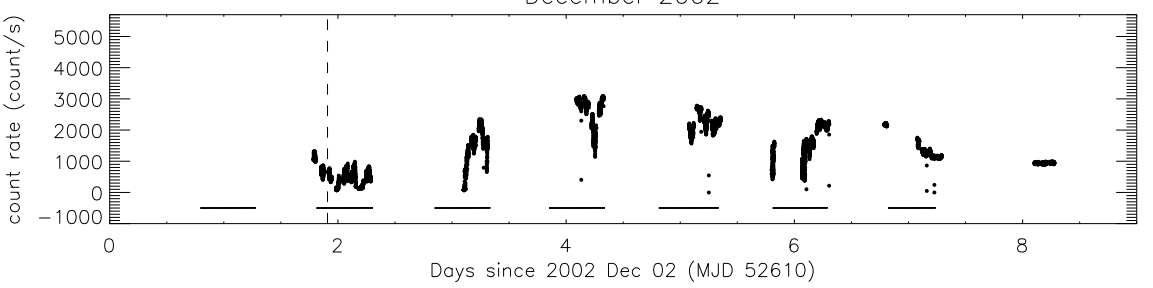

Figure 1: 2-18 keV RXTE/PCA light curves for the two data sets examined here. The bin size is 16 seconds. Only counts from PCU2 were used. Horizontal lines in the bottom part of each light curve correspond to ATCA radio observations, vertical dashed lines correspond to phase 0.0 of the orbital period, vertical dotted lines correspond to phase 0.5 .

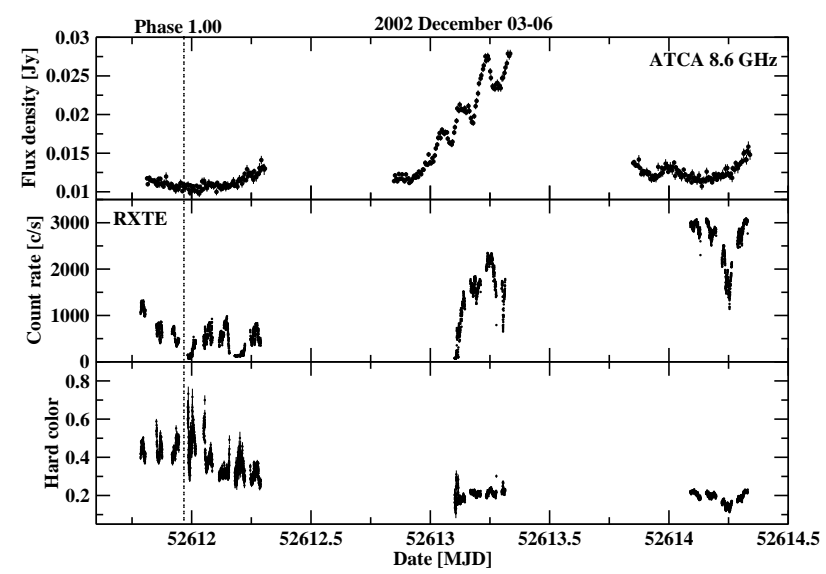

Figure 2: Radio and X-ray light curves (top and middle panels) and X-ray hardness curve (bottom panel) for 2002 December 03-06, between phase 0.99 and 1.15 of the orbital period. Multiple radio flares are clearly visible after the passage through the periastron

not immediately) a multiple-flare event was clearly detected in radio (both at 4.8 and $8.6 \mathrm{GHz}$ ), simultaneous with a sudden increase of the X-ray flux and variations in the X-ray hardness. In Figure 3 (left panel) we present a X-ray HID where points corresponding to different days are plotted in different colours: the track drawn by the source in the HID changes its position and morphology after the passage through phase 0.0 of the orbital phase, passing from a "cloud" located in the left-side of the diagram to a series of horizontal "strips" in the bottom side. The transition happens on December 5 (third HID in Figure 3 left panel, red points), although it is not sharp: 

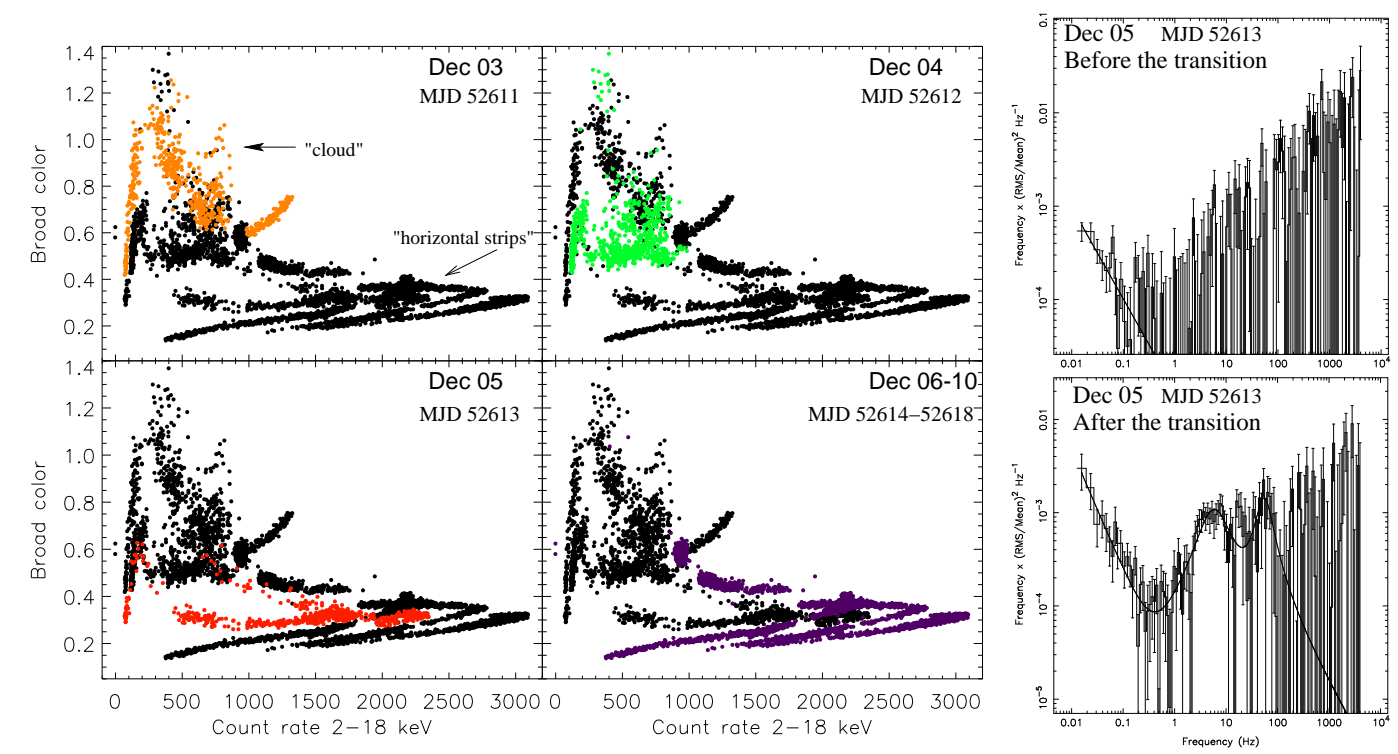

Figure 3: Left panel: Hardness-intensity diagram for 2002 December. The bin size is 16 seconds. In each panel, points corresponding to specific orbital intervals are plotted in different colours. "Cloud" and "horizontal strips" refer to specific zones of the HID (see the text). Right panel: power density spectra averaged before (top spectrum) and after (bottom spectrum) the spectral transition around phase 0.0. Spectra before/after the transition have not been averaged on the whole cloud/strips region respectively, but on a smaller selection of points (details will be given in Soleri et al., in preparation). The continuous line represents the best fit to the data. Spectra are plotted in the $v P_{v}$ representation [1].

a transition cloud-strips occurs, followed after approximately 5 hours by a rapid transition (about 200 s) strips-cloud-strips.

Power density spectra averaged in the HID before and right after the X-ray spectral transition are shown in Figure 3 (right panel). Their properties change considerably: the spectrum before the transition is characterized by a weak VLFN ( $r m s=3.15 \pm 0.18 \%$ integrated in the range $0.01-100$ $\mathrm{Hz})$ while after the transition the $r m s$ of the VLFN increases $(6.06 \pm 0.58 \%$ in the same range) and a normal branch oscillation appears $(v=5.87 \pm 0.62 \mathrm{~Hz}, r m s=4.48 \pm 0.28 \%)$.

\subsection{October - Phase 0.5 of the orbital period}

Figure 4 shows the radio light curve, the X-ray light curve and the X-ray hardness curve for the orbital phase interval $0.40-0.55$, in 2000 October. From the radio light curve, a sequence of radio flares nearby the passage through the apastron is evident. Radio flares close to this phase are peculiar and unexpected with respect to what can be found in the literature where significant radio flux density enhancements are associated just to phase 0.0 (Tudose et al., in preparation). To date there is only one claim of a radio flare associated with phase 0.5 [7].

To test whether this sequence is associated with any spectral change in the X-rays, in Figure 5 (left panel) we show a "zoom" of Figure $\bigoplus$ corresponding to October 09-10 and the hardness-intensity diagram for all 2000 October (right panel). Purple and blue points in the HID correspond to the two portions of the light curve in the left panel and are used to mark two different spectral branches. Since a classification of the spectral branches in the hardness-intensity diagram is beyond the scope 


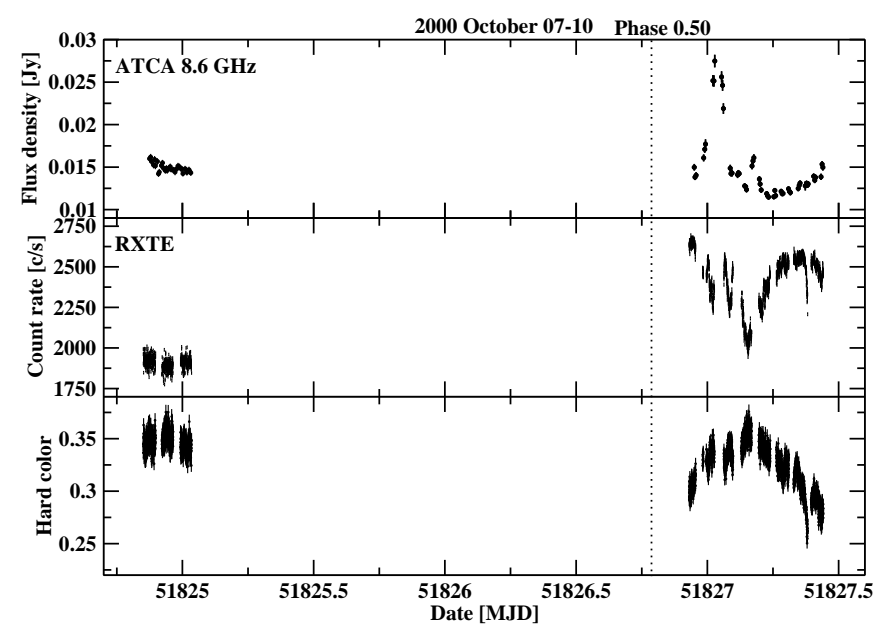

Figure 4: Radio and X-ray light curves (top and middle panels) and X-ray hardness curve (bottom panel) for 2000 October $07-10$, between phase 0.40 and 0.55 of the orbital period. Multiple radio flares are clearly visible close to phase 0.5
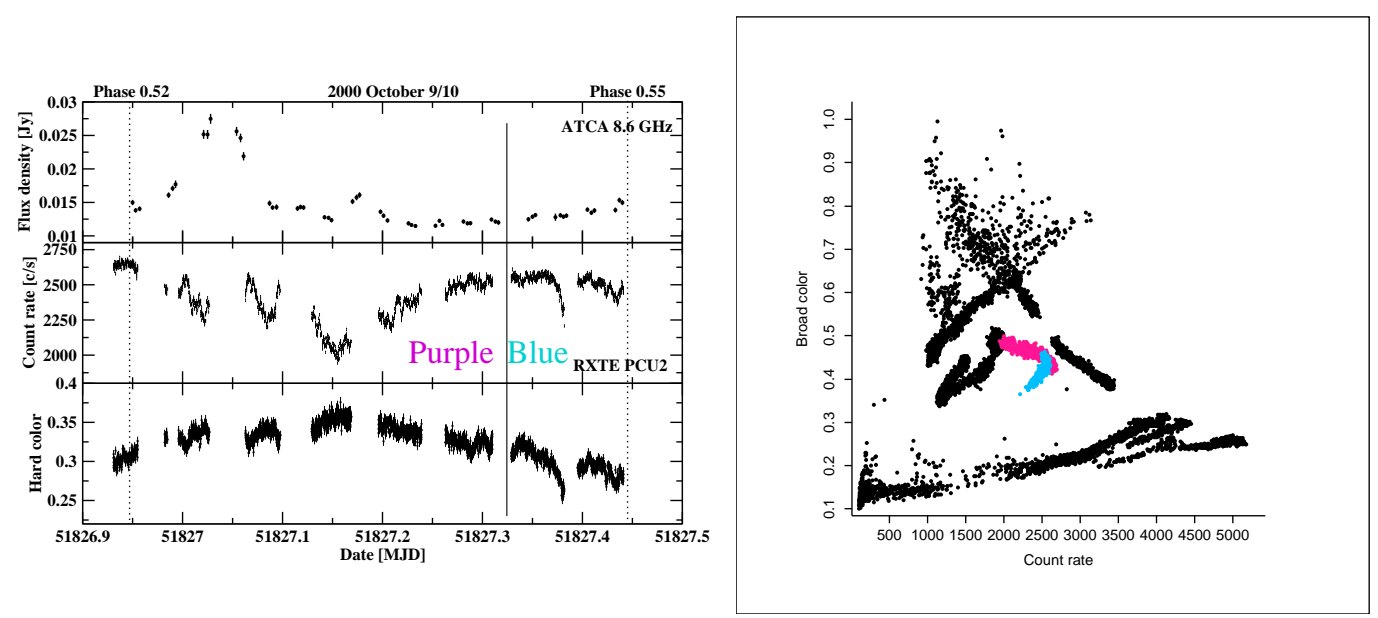

Figure 5: Left panel: zoom of Figure 4 around 2000 October 09-10; Right panel: HID for 2000 October. Different colours in the HID correspond to different sides (in respect to the vertical line) of the X-ray light curve

of this paper, for now we will assert without demonstration that the purple points track a $\mathrm{HB}$ and the blue points a NB. A discussion will be presented in a future paper (Soleri et al., in preparation). On 2000 October 09-10 RXTE clearly caught a spectral transition between the HB and the NB, however this clearly occurred after the radio flare.

\section{Conclusions}

We have analysed simultaneous X-ray/radio observations of Cir X-1 obtained with RXTE and 
ATCA respectively.

Phase 0.0: On 2002 December 05, near periastron passage, we detected a multiple radio flare event, corresponding to a fast change of the X-ray spectral/timing properties (in $\$ 3.1$ we already remarked that in the HID the transition is not sharp). Our detection could be the first direct observation of a radio flare associated with a spectral transition in the X-ray band in a NS system, since no clear association between X-ray spectral/timing changes and the emission of a radio flare has been reported yet. Recently it has been suggested that in NS-Z sources the spectral transitions from the HB to the NB might be the moment of the launch of transient radio jets [21], by analogy with what is suggested for BHCs, where the hard intermediate state to soft intermediate state transition might drive the ejection of transient radio plasmons [4], [10]. In BHCs these state transitions have been in turn associated with the presence, in the PDS, of a transient low-frequency QPO, the so called type-B [2]. An association between the type-B QPO detected in BHCs and the normal branch oscillation observed in $\mathrm{Z}$ sources has been suggested in [5], since these QPOs present similar properties: on 2002 December we could associate the radio flare to a X-ray spectral transition that is in turn associated with the presence of a normal branch oscillation, although we do not see any obvious HB to NB passage.

Phase 0.5: On 2000 October 09 we detected a radio flare near the passage through the apastron. In the literature there is only circumstantial evidence for radio flares at phase 0.5 [7], maybe partly because the observing strategies concentrated around phase 0.0. This is the first clear evidence of a radio flare near phase 0.5 (Tudose et al., in preparation). In the RXTE data we also detected a spectral transition on 2000 October 09 about 2.5 hours after the end of the second radio flare, with the source moving from the HB to the NB. As it has been suggested in [21], this transition might be the moment of the launch of transient radio jets, but in our specific case the time difference between the two events does not allow us to associate them. Unluckily we do not have RXTE data right before the beginning of the radio flaring activity (see Figure $\emptyset$ ).

\section{References}

[1] Belloni, T., Psaltis, D., van der Klis, M., 2002, ApJ, 572, 392

[2] Belloni, T., Homan, J., Casella, P., van der Klis, M., Nespoli, E., Lewin, W. H. G., Miller, J. M., Méndez, M., 2005, A\&A, 440, 207

[3] Boutloukos, S., van der Klis, M., Altamirano, D., Klein-Wolt, M, Wijnands, R., Jonker, P. G., Fender, R. P., 2006, ApJ, 653, 1435

[4] Casella, P., Belloni, T., Homan, J., Stella, L., 2004, A\&A, 426, 587

[5] Casella, P., Belloni, T., Stella, L., 2005, ApJ, 629, 403

[6] Ding, G. Q., Qu, J. L., Li, T. P., 2003, Astr. J., 596, L219

[7] Fender, R. P., 1997, Proceedings of the Fourth Compton Symposium, ed. Dermer, C. D., Strickman, M. S, Kurfess, J. D., p. 798

[8] Fender, R., Spencer, R., Tzioumis, T., Wu, K., van der Klis, M., van Paradijs, J., Johnston, H., 1998, ApJL, 506, L121

[9] Fender, R., Wu, K., Johnston, H., Tzioumis, T., Jonker, P., Spencer, R., van der Klis M., 2004, Nat, 427, 222 
[10] Fender, R. P., Belloni, T. M., Gallo, E., 2004, MNRAS, 355, 1105

[11] Fomalont, E. B., Geldzahler, B. J., Bradshaw, C. F., 2001, ApJ, 559, 283

[12] Glass, I. S., 1978, MNRAS, 183, 335

[13] Hasinger, G. \& van der Klis, M., 1989, A\&A, 225, 79

[14] Haynes, R. F., Jauncey, D. L., Murdin, P. G., Goss, W. M., Longmore, A. J., Simons, L. W. J., Milne, D. K., Skellern, D. J., 1978, MNRAS, 185, 661

[15] Heinz, S., Schulz, N. S., Brandt, W. N., Galloway, D. K., 2007, ApJL, 663, L93

[16] Homan, J., van der Klis, M., Wijnands, R., Belloni, T., Fender, R., Klein-Wolt, M., Casella, P., Méndez, M., Gallo, E., Lewin, W. H. G, Gehrels, N., 2007a, ApJ, 656, 420

[17] Homan, J., Wijnands, R., Altamirano, D., Belloni, T., 2007b, ATEL, 1165, 1

[18] Jonker, P. G. \& Nelemans, G., 2004, MNRAS, 354, 355

[19] Kaluzienski, L. J., Holt, S. S., Boldt, E. A., Serlemitsos, P. J., 1976, ApJL, 208, L71

[20] Margon, B., Lampton, M., Bowyer, S., Cruddace, R., 1971, ApJL, 169, L23

[21] Migliari, S. \& Fender, R. P, 2006, MNRAS, 366, 79

[22] Moneti, A., 1992, A\&A, 260, L7

[23] Murdin, P, Jauncey, D. L., Lerche, I., Nicolson, G. D., Kaluzienski, L. J., Holt, S. S., Haynes, R. F., 1980, A\&A, 87, 292

[24] Nicolson, G. D., Glass, I. S., Feast, M. W., 1980, MNRAS, 191, 293

[25] Nicolson, G. D., 2007, ATEL, 985, 1

[26] Oosterbroek, T., van der Klis, M., Kuulkers, E., van Paradijs, J., Lewin, W., 1995, A\&A, 297, 141

[27] Penninx, W., Lewin, W. H. G., Zijlstra, A. A., Mitsuda, K., van Paradijs J., 1988, Nat, 336, 146

[28] Psaltis, D., Belloni, T., van der Klis, M., 1999, ApJ, 520, 262

[29] Shirey, R. E., Bradt, H. V., Levine, A. M., Morgan, E. H., 1998, ApJ, 506, 374

[30] Shirey, R. E., Bradt, H. V., Levine, A. M., 1999, ApJ, 517, 472

[31] Tan, J., Lewin, W. H. G., Hjellming, R. M., Penninx, W., van Paradijs, J., van der Klis, M., Mitsuda, K., 1992, ApJ, 385, 314

[32] Tennant, A. F., Fabian, A. C., Shafer, R. A., 1986, MNRAS, 221, 27P

[33] Tudose, V., Fender, R., Kaiser, C., Tzioumis, A., van der Klis, M., Spencer R., 2006, MNRAS, 372, 417

[34] van der Klis, M., 2006, in "Compact Stellar X-ray Sources”, ed. W. H. G. Lewin \& M. van der Klis, Cambridge University Press, Cambridge, p. 39

[35] Wijnands, R. A. D., van der Klis, M., Psaltis, D., Lamb, F. K., Kuulkers, E., Dieters, S., van Paradijs, J., Lewin, W. H. G., 1996, ApJL, 469, L5

[36] Wijnands, R. A. D., van der Klis, M., Kuulkers, E., Asai, K., Hasinger, G., 1997, A\&A, 323, 399 